\title{
From Corporate Memory to Corporate Knowledge
}

\author{
Dennis Tsichritzis \\ Ricoh, Research and Development Group \\ 2, Route de Florissant 1206 Geneva, Switzerland \\ dennis.tsichritzis@gmail.com
}

\begin{abstract}
Technology enables us to store and recuperate with a Google like interface all documents produced within the boundaries of a company. Does this mean we have complete Corporate Memory? Yes and No because there are problems to be solved in Accountability, Authority and Responsibility. This is not a free for all environment where people can co-create material of nonuniform quality and value. We are also far for having Corporate Knowledge since the rules, the processes, the acceptable scenarios, the past lessons and the successful cases are missing. We will discuss what type of environment will be needed and outline what problems need to be solved. This talk does not represent in any way the positions of Ricoh as a company and should be considered as an individual opinion.
\end{abstract}

